\title{
Conjunctival Inflammatory Nodule in a Patient with Pityriasis Lichenoides et Varioliformis Acuta
}

\author{
Elad Moisseiev David Varssano \\ Department of Ophthalmology, Tel Aviv Sourasky Medical Center, Tel Aviv, Israel
}

\section{Key Words}

Pityriasis lichenoides · Inflammation · Nodule

\begin{abstract}
Aim: To report a case of a patient with pityriasis lichenoides et varioliformis acuta (PLEVA) who developed a conjunctival inflammatory nodule.

Case Report: a 13-year-old boy with a diffuse papulovesicular rash diagnosed as PLEVA presented to our clinic complaining of irritation in his right eye. Slitlamp examination revealed a conjunctival inflammatory nodule. His symptoms resolved under topical steroid treatment.

Discussion: Pityriasis lichenoides (PL) is a rare condition, diagnosed by a combination of a typical clinical presentation and histopathology. Mucous membrane involvement has been described in severe cases. Ocular involvement in PL has seldom been reported. We suggest that conjunctival inflammatory nodules may be part of the clinical spectrum of this condition and should be looked for and treated in these patients.
\end{abstract}

\section{Introduction}

Pityriasis lichenoides (PL) is an uncommon acquired skin condition, which is challenging to diagnose and treat [1]. It tends to occur in late childhood and is more common in males [2]. The disease is clinically characterized by polymorphic papulosquamous dermatitis, and histologically by interface dermatitis with prominent lymphocytic infiltration [1]. In severe cases, there may be mucous membrane involvement [3]. PL has a spectrum consisting of 3 overlapping sub-types: pityriasis lichenoides et varioliformis acuta (PLEVA), pityriasis lichenoides chronica (PLC), and febrile ulceronecrotic Mucha-Habermann disease (FUMHD). PLEVA presents as an acute eruption of multiple erythematous papules, PLC has a milder and more gradual course, and FUMHD is the most severe form, presenting as an acute, severe, generalized 
eruption of purpuric and ulceronecrotic plaques, with fever, systemic involvement and up to $25 \%$ mortality [1].

\section{Case Report}

We report a 13-year-old boy who presented to our clinic complaining of redness and irritation of his right eye. 3 weeks prior to his complaint, he had developed a papulovesicular rash with multiple lesions involving his face, neck, trunk and extremities. He also suffered from perioral involvement and mouth ulcers. There was no fever. The patient had a 7-year history of benign rolandic epilepsy treated with carbamazepine. There were no recent seizures and no change in dosage. Six years prior to his presentation, he had developed pallor of the right eyelids and poliosis, which were diagnosed as vitiligo ( fig. 1). Renal and liver function tests were normal, erythrocyte sedimentation (ESR) rate and C-reactive protein (CRP) levels were within normal limits, and complete blood count was normal except for mild eosinophilia. Serologic tests for active infection by cytomegalovirus (CMV), Epstein-Barr virus (EBV), coxsackievirus, parvovirus, enterovirus, mycoplasma, toxoplasma, Rickettsia and antistreptolysin $\mathrm{O}$ were all negative. As drug hypersensitivity immune reaction was initially suspected, he was initially treated with cessation of carbamazepine treatment as well as oral cephalexin, antihistamines and both oral and topical steroidal treatment. Skin biopsy of one of the lesions demonstrated interface dermatitis, diffuse perivascular dermal lymphocytic infiltration, epidermal lymphocytic infiltration, epidermal pallor and parakeratosis (fig. 2). Diagnosis of PLEVA was established. The treatment regimen was changed to oral erythromycin and topical steroids.

Upon presentation at our clinic, visual acuity was 20/20 OU. There were bilateral eyelid skin lesions, but no involvement of the palpebral conjunctiva on eversion. Observation of the conjunctiva of the right eye revealed an erythematous nodule at the inferotemporal quadrant, adjacent to the limbus (fig. 3 ). The cornea was clear and uninvolved. The anterior segment and fundus were otherwise normai. Examination of the left eye was completely normal. The patient was treated with topical steroids, and the nodule resolved after 2 weeks.

\section{Discussion}

The incidence and prevalence of PL are unclear. Its incidence has been estimated to be approximately 1 in 2,000 people [4]. Fortunately, the severe FUMHD is extremely rare [3]. The pathogenesis of the disease is debated in the literature and has been hypothesized to be either an autoimmune reactive process or a T-lymphocyte premalignant disorder [1].

Ocular involvement in any of the three PL subtypes has rarely been reported. Suarez et al. [5] reported conjunctival ulceration in a patient with FUMHD, and Verhamme et al. [6] reported bilateral conjunctival inflammatory nodules in a patient with PLEVA.

Our patient presented with a conjunctival inflammatory nodule resembling the only previous ocular manifestation described in PLEVA [6]. We believe our report reinforces this finding as a part of the variable spectrum of the clinical presentation of this disease. We suggest that all patients with PL, especially if mucosal involvement is present, should undergo an ophthalmologic examination as part of their evaluation. In addition, we stress the fact that the patient had previously developed vitiligo, which suits the hypothesis that $\mathrm{PL}$ is autoimmune in nature and strengthens its association with other autoimmune disorders $[1,7]$. 
Fig. 1. Previous poliosis and vitiligo of the right eyelids. Multiple papulosquamous lesions are seen on and around the eyelids.

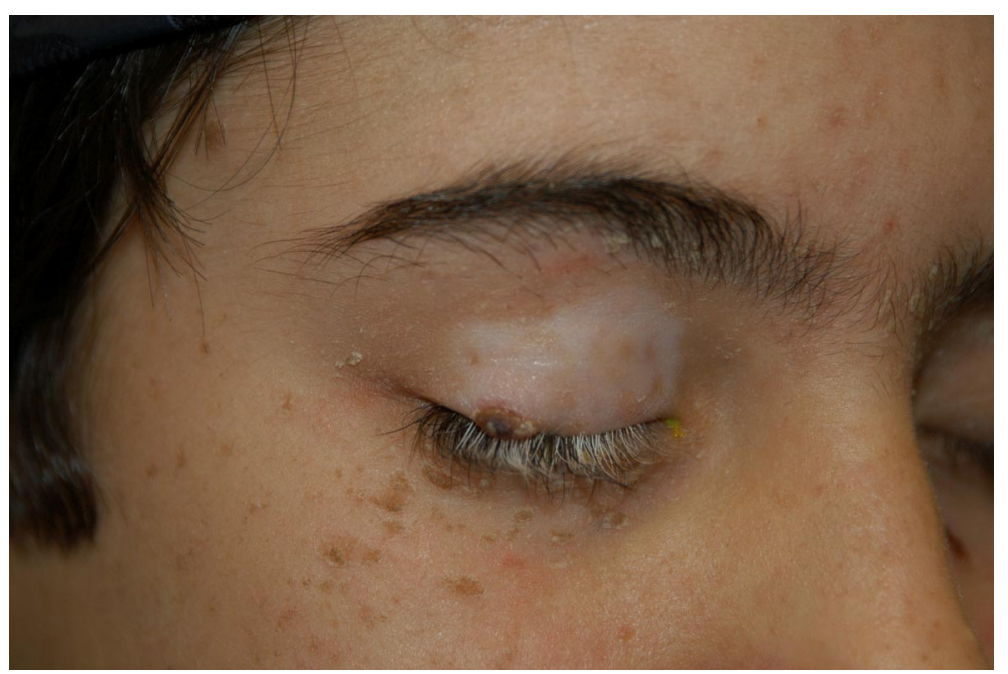

Fig. 2. Biopsy demonstrating interface dermatitis, diffuse perivascular dermal lymphocytic infiltration, epidermal lymphocytic infiltration, epidermal pallor and parakeratosis, consistent with PLEVA.

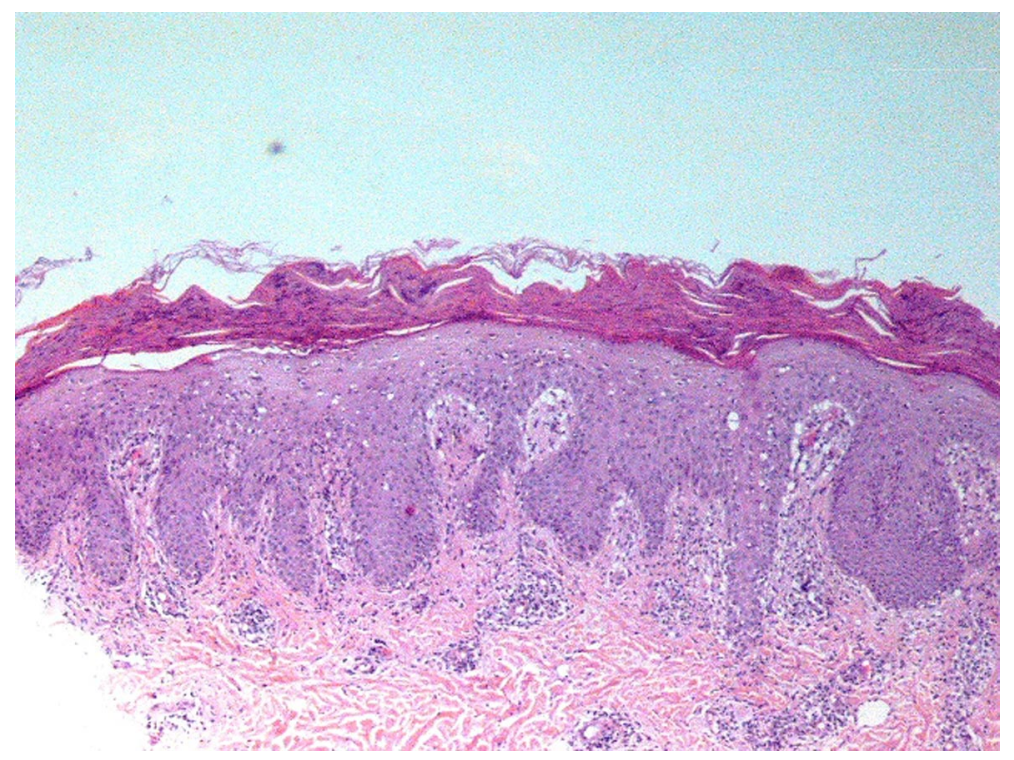


Fig. 3. Conjunctival inflammatory nodule in the inferotemporal quadrant of the right eye.

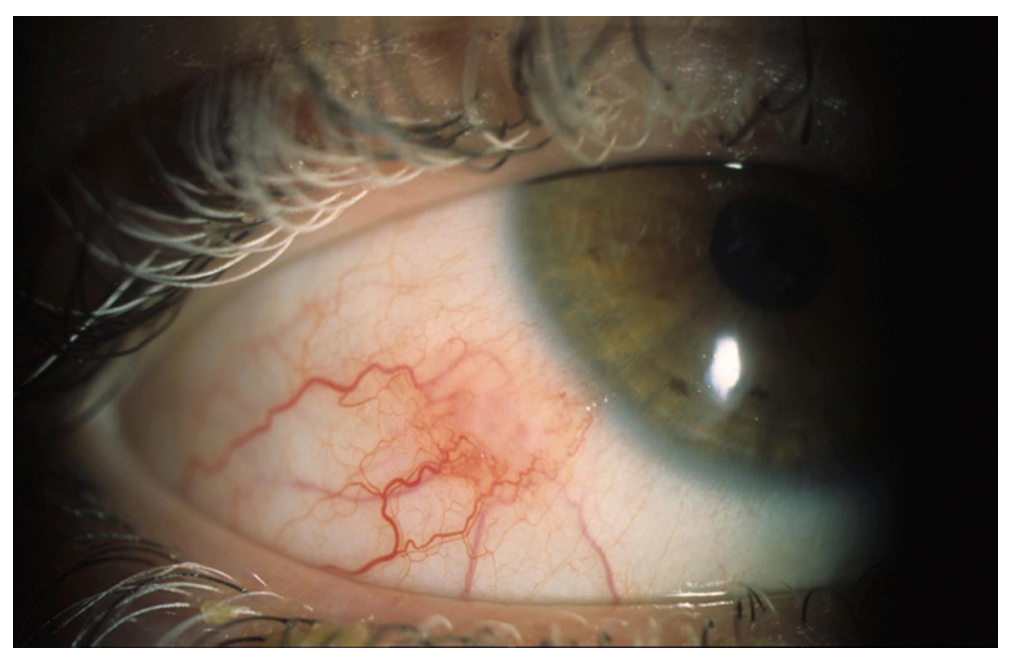




\section{References}

1 Khachemoune A, Blyumin ML: Pityriasis lichenoides: pathophysiology, classification, and treatment. Am J Clin Dermatol 2007;8:29-36.

2 Wood GS, Reizner G: Other papulosquamous disorders; in Bologna JL, Jorizzo J, Rapini R (eds): Dermatology. London, CV Mosby, 2003, pp 151-163.

3 Bowers S, Warshaw EM: Pityriasis lichenoides and its subtypes. J Am Acad Dermatol 2006;55:557-572.

4 Daoud MS, Pittelkow MR: Pityriasis lichenoides; in Freeberg IM, Eisen AZ, Wolff $\mathrm{K}$, et al. (eds): Fitzpatrick's Dermatology in General Medicine. 6th ed. New York, McGraw-Hill Inc., 2003, pp 456-462.

5 Suarez J, Lopez B, Villalba R, et al: Febrile ulceronecrotic Mucha-Habermann disease: a case report and review of the literature. Dermatology 1996;192:277-279.

-6 Verhamme T, Arnaout A, Ayliffe WH: Limbal and bulbar inflammatory nodules in a patient with pityriasis lichenoides et varioliformis acuta. Bull Soc Belge Ophthalmol 2008;307:13-18.

7 Tsuji T, Kasamatsu M, Yokota M, et al: Mucha-Habermann disease and its febrile ulceronecrotic variant. Cutis 1996;58:123-131. 